\title{
The Goldilocks technique: An alternative method to construct a breast mound after prosthetic breast reconstruction failure
}

\author{
Karina Jaikel Zavala, Jin Geun Kwon, Hyun Ho Han, Eun Key Kim, Jin Sup Eom \\ Department of Plastic Surgery, Asan Medical Center, University of Ulsan College of Medicine, Seoul, Korea
}

The Goldilocks technique for breast reconstruction utilizes redundant mastectomy flap tissue to construct a breast mound. This technique is suitable for women who decline, or are poor candidates for, traditional postmastectomy reconstruction. Moreover, this technique can be applied in secondary operations after the failure of initial reconstruction efforts. A 74-yearold patient underwent the Goldilocks procedure after reconstruction failure with an implant and acellular dermal matrix. At her 6-month follow-up, the cosmetic outcome of the procedure was satisfactory, and no complications were noted. Therefore, the Goldilocks procedure is a safe alternative to reconstruct breast mounds following reconstruction failure, especially in obese patients.

Keywords Mammaplasty / Breast implants / Acellular dermis / Seroma

\author{
Correspondence: Hyun Ho Han \\ Department of Plastic Surgery, Asan \\ Medical Center, University of Ulsan \\ College of Medicine, 88 Olympic-ro \\ 43-gil, Songpa-gu, Seoul 05505, \\ Korea \\ Tel: +82-2-3010-3600 \\ Fax: +82-2-476-7471 \\ E-mail: tripleh1952@gmail.com
}

\section{INTRODUCTION}

In the past few years, skin-sparing mastectomy has become an extremely popular and oncologically safe option for women with breast cancer. Currently, there are several different approaches for reconstruction using prosthetic implants and autologous tissues, each with its own indications, related complications, advantages, and disadvantages [1].

Peri-implant seroma is a common complication of breast prosthetic reconstruction and mammoplasty [2]. Various causes of late seroma development have been reported, including infection, implant rupture, mechanical shearing, and breast implantassociated anaplastic large cell lymphoma [2].

After acellular dermal matrix (ADM) was introduced for breast reconstruction procedures in 2001, the combination of nipple- and skin-sparing mastectomy and immediate breast reconstruction with $\mathrm{ADM}$ and submuscular implant placement has become significantly more common [3]. According to the existing literature, $\mathrm{ADM}$ is associated with an increased risk of seroma. In a meta-analysis, the rate of seroma formation after implant-based breast reconstruction using $\mathrm{ADM}$ was $4.8 \%$ to $6.9 \%$ [3]. However, information on the incidence of seroma formation remains limited, warranting standardized reporting [3].

Richardson and $\mathrm{Ma}$ [4] described the Goldilocks mastectomy technique in 2012. This technique utilizes the redundant inferior mastectomy flap tissue to construct a breast mound. After skin-sparing mastectomy, the de-epithelialized mastectomy flap within the boundary of the Wise pattern incision is molded to produce the breast volume [4]. This technique has been traditionally used for breast reconstruction in women who are poor 
candidates for conventional methods due to increased risks associated with obesity or medical comorbidities and in those who decline a complicated reconstruction procedure and focus on simplifying the treatment for individual reasons [4-7]. The ideal patients for this technique include women who are candidates for skin-sparing mastectomy and have macromastia or significant breast ptosis [5-9].

Moreover, the Goldilocks technique could be applied as a salvage technique following the failure of initial reconstruction. If breast reconstruction is not successfully performed with a prosthetic implant, this technique could be used to construct a breast mound and prepare the tissue for secondary surgery. In this report, we present the case of a 74-year-old woman with a history of failure of postmastectomy immediate breast reconstruction with an implant and ADM. For patients who suffer from prolonged seroma, radical capsulectomy with breast reconstruction using autologous tissue, such as a deep inferior epigastric artery perforator free flap, is preferred as a treatment choice at our center. However, this patient was elderly, and did not want to undergo a complicated reconstruction procedure. Considering the patient's needs, the Goldilocks technique was planned.

\section{CASE}

A 74-year-old woman underwent nipple- and skin-sparing mastectomy due to breast cancer (stage 1A, T1NOM0) and immediate breast reconstruction using an implant $(280 \mathrm{~mL}$, anatomic, Allergan Natrelle) and ADM (Megaderm) at another local hospital. The patient presented to Asan Medical Center to resolve a seroma that had lasted for 5 months postoperatively. Her physical examination revealed no evident signs of breast infec- tion, but fluctuation due to the presence of seroma was noted (Fig. 1). The seroma had to be drained every 3 days, with 150 $200 \mathrm{~mL}$ of fluid drained at each aspiration. Limited information was available about the previous operation. As a treatment option, we suggested breast reconstruction with autologous tissue, such as abdominal tissue or the latissimus dorsi muscle. However, the patient decided to have the implant removed and did not wish to replace it.

The dead space that resulted from a volume mismatch between the large, ptotic breast envelope and the small implant seemed to have been the main cause of the seroma. To decrease the volume mismatch, the authors recommended the Goldilocks technique to change the mastectomy flap into a spacer replacing the implant, and the patient agreed to this strategy. Thereafter, a Wise pattern design and incision were performed (Fig. 2A). The implant was placed in the pre-pectoral plane and the seroma with frayed ADM was intraoperatively noted (Fig. 2B). There were no lymphatic openings or infectious signs. Because there was substantial subcutaneous tissue at the mastectomy flap, capsulectomy was performed to minimize the risk of seroma recurrence. The inferior mastectomy flap was de-epithelialized and used to form the bulk of the reconstructed breast mound in a rolling manner, from the distal to cephalic edge. The location of the inferior mastectomy flap was determined with the patient in the seated position, and 3-0 Vicryl sutures were placed superiorly through the molded flap at the pectoralis major muscle (Fig. 2C).

The nipple-areolar complex was located within the area that required de-epithelization, so the nipple was grafted after completing skin closure. A contralateral breast reduction procedure using the Wise pattern and McKissock bipedicle technique was performed to reduce the size discrepancy by removing $330 \mathrm{~g}$ of
Fig. 1. Preoperative evaluation

(A) A 74-year-old patient who presented with chronic seroma underwent nipple- and skin-sparing mastectomy and immediate reconstruction with an implant and acellular dermal matrix. (B) Computed tomography showing a large amount of liquid around the implant in the breast pocket.
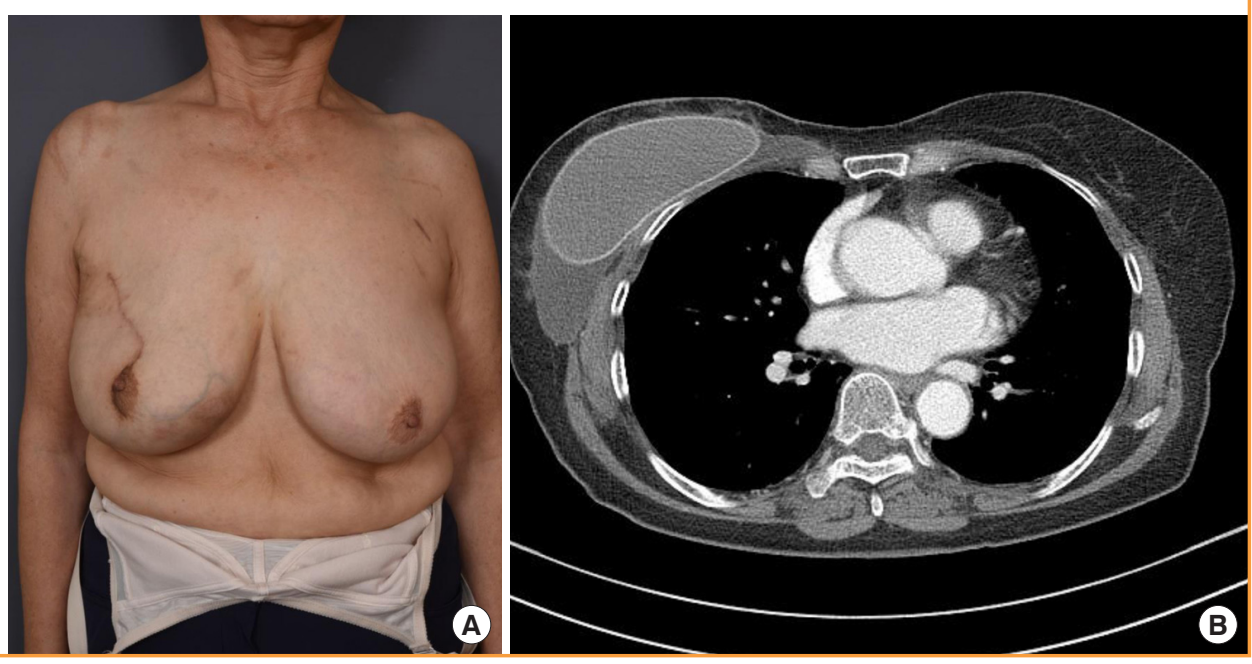
tissue. No evidence of postoperative complications was noted (Fig. 3A). The surgical outcome at a 6-month follow-up is presented in Fig. 3B. Although the patient was offered an implant placement and tattooing following complete wound healing to obtain superior symmetry and better cosmetic outcomes, she declined it for individual reasons.

\section{DISCUSSION}

Postmastectomy reconstruction is typically performed using implant-based techniques $[1,5,10,11]$. Although the majority of patients have a completely uneventful postoperative course, unfortunately, some patients experience complications [12], one of the most common of which is seroma development [12]. To enhance the outcomes of implant-based breast reconstruction, $\mathrm{ADM}$ - a durable, nonimmunogenic, and elastic material) derived from human or porcine skin tissue - was introduced to reinforce the pocket for the implant; however, a disadvantage of $\mathrm{ADM}$ is that it is associated with a higher rate of seroma formation [3]. In a previous meta-analysis [10], $4.8 \%$ of patients developed seroma after reconstruction with human $\mathrm{ADM}$, whereas $3.5 \%$ of patients developed seroma after reconstruction with submuscular prostheses. Another meta-analysis [3] reported that approximately $5 \%$ of the included patients developed seroma when $\mathrm{ADM}$ was used for nipple-sparing mastectomy and immediate breast reconstruction, but pointed out that reliable

\section{Fig. 2. Preoperative plan and intraoperative photograph}

(A) The schematic of the preoperative plan for incisions, free nipple, and contralateral breast reduction. (B) Seroma and frayed acellular dermal matrix (ADM) were noted intraoperatively. (C) Intraoperative photograph after implant and ADM removal and de-epithelialization of the inferior skin flap. The Goldilocks-method flap was superiorly folded to construct a new breast mound.
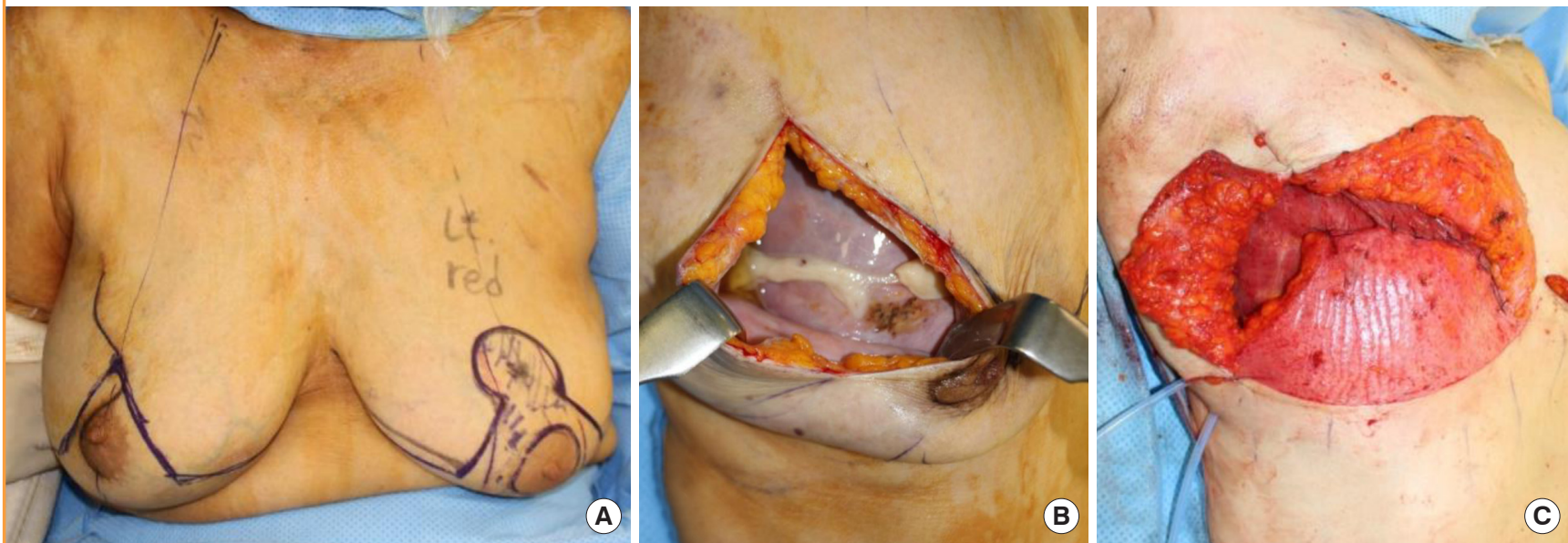

Fig. 3. Immediate and 6-month postoperative photographs

(A) A contralateral breast reduction using the Wise pattern and McKissock bipedicle technique was performed to achieve symmetry. (B) Sixmonth postoperative photography showing no complications during the follow-up. The patient refused any further procedures.
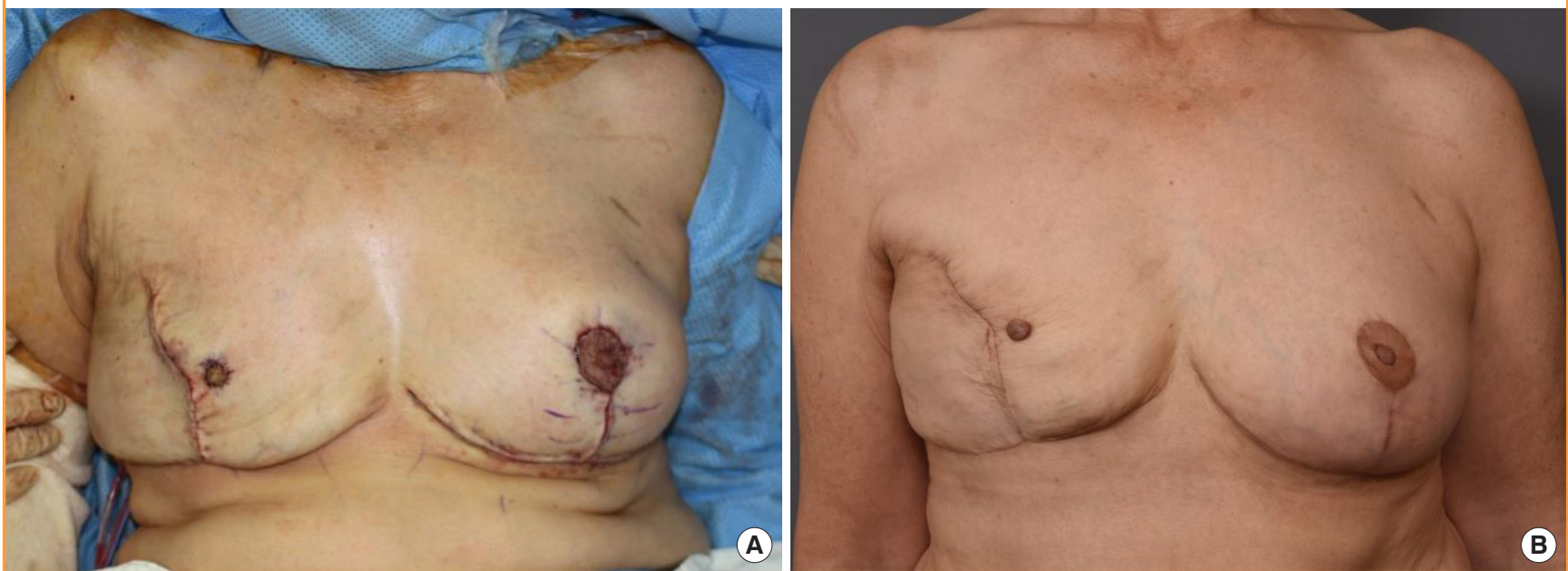
scientific evidence regarding complications after this technique is limited in the existing literature.

The Goldilocks procedure is a breast reconstruction option for women who have contraindications for complex reconstruction procedures or who decline the conventional procedures [46]. This technique includes the use of de-epithelialized inferior pole skin flaps as local autologous dermal-cutaneous flaps to reconstruct a breast mound following skin-sparing mastectomy $[4,5]$. In the mastectomy procedure, an inverted $\mathrm{T}$ approach is applied, and it is important to retain the subcutaneous fat thickness in the area distant from the tumor $[7,9]$. The de-epithelialized tissue is positioned on the chest wall, and the skin incisions are closed $[4,7]$.

In previous reports $[4,9]$, this technique was generally used for reducing complications such as skin necrosis of the mastectomy flap of a ptotic and large breast $[4,9]$. However, this procedure is also suitable as a salvage technique in patients with implant-induced complications, as in the present case report. After stabilization of the condition of the breast, surgeons have the opportunity to use classic reconstruction options such as fat grafting injections and prosthetic implants if the patient wishes to undergo a procedure at a high-volume mastectomy site and has no medical contraindications $[4,9]$. If the patient refuses an additional procedure and focuses on simplifying the treatment and follow-up process, as in our case, the Goldilocks procedure alone can maintain the shape of the breast, although with a size consistent with an extreme breast reduction [4]. However, for optimal outcomes, this technique should be limited to obese patients with large, ptotic breasts in order to obtain sufficient tissue volume.

In conclusion, the Goldilocks procedure seems promising as a safe alternative to reconstruct breast mounds in obese patients with primary failure following a breast reconstruction procedure.

\section{NOTES}

\section{Conflict of interest}

No potential conflict of interest relevant to this article was reported.

\section{Ethical approval}

The study was approved by the Institutional Review Board of Asan Medical Center (IRB No. S2019-0773-0001) and performed in accordance with the principles of the Declaration of Helsinki. Written informed consent was obtained.

\section{Patient consent}

The patient provided written informed consent for the publication and the use of her images.

\section{Author contribution}

Data collection: Zavala KJ. Writing - original draft: Zavala KJ, Kwon JG, Han HH. Perform the procedures: Han HH. Technical support as a study supervisor: Kim EK, Eom JS.

\section{ORCID}

Karina Jaikel Zavala https://orcid.org/0000-0003-3769-4336

Jin Geun Kwon https://orcid.org/0000-0002-5873-3077

Hyun Ho Han https://orcid.org/0000-0001-7072-9882

Eun Key Kim https://orcid.org/0000-0002-3986-6886

Jin Sup Eom https://orcid.org/0000-0003-3229-2012

\section{REFERENCES}

1. Bletsis P, Bucknor A, Chattha A, et al. Evaluation of contralateral and bilateral prophylactic mastectomy and reconstruction outcomes: comparing alloplastic and autologous reconstruction. Ann Plast Surg 2018;80:S144-9.

2. Di Napoli A, Pepe G, Giarnieri E, et al. Cytological diagnostic features of late breast implant seromas: from reactive to anaplastic large cell lymphoma. PLoS One 2017;12:e0181097.

3. Heidemann LN, Gunnarsson GL, Salzberg CA, et al. Complications following nipple-sparing mastectomy and immediate acellular dermal matrix implant-based breast reconstruction-a systematic review and meta-analysis. Plast Reconstr Surg Glob Open 2018;6:e1625.

4. Richardson H, Ma G. The Goldilocks mastectomy. Int J Surg 2012;10:522-6.

5. Schwartz JC. Goldilocks mastectomy: a safe bridge to implant-based breast reconstruction in the morbidly obese. Plast Reconstr Surg Glob Open 2017;5:e1398.

6. Ter Louw RP, Bruce SB, Nahabedian MY. Partial breast reconstruction with goldilocks technique after excision of giant fibroadenoma: a case report. Plast Reconstr Surg Glob Open 2017;5:e1200.

7. Ogawa T. Goldilocks mastectomy for obese Japanese females with breast ptosis. Asian J Surg 2015;38:232-5.

8. Schwartz JC, Skowronski PP. Case report of a definitive autologous reconstruction in a patient requiring immediate postoperative anticoagulation and reduced operative time. Int J Surg Case Rep 2016;25:86-8.

9. Schwartz JD, Skowronksi PP. Extending the indications for autologous breast reconstruction using a two-stage modified Goldilocks procedure: a case report. Breast J 2017;23:344-7. 
10. Kim JY, Davila AA, Persing S, et al. A meta-analysis of human acellular dermis and submuscular tissue expander breast reconstruction. Plast Reconstr Surg 2012;129:28-41.

11. Weinstein B, Kumar A, Smith P, et al. Moffitt cancer center experience of tissue expander breast reconstruction: does acellular dermal matrix increase return to the operating room? Ann Plast Surg 2018;80:S377-80.

12. Hatem J, Tobey JD, Solomon MP, et al. FNA of peri-implant seroma of the breast. Cytopathology 2018;29:300-2. 